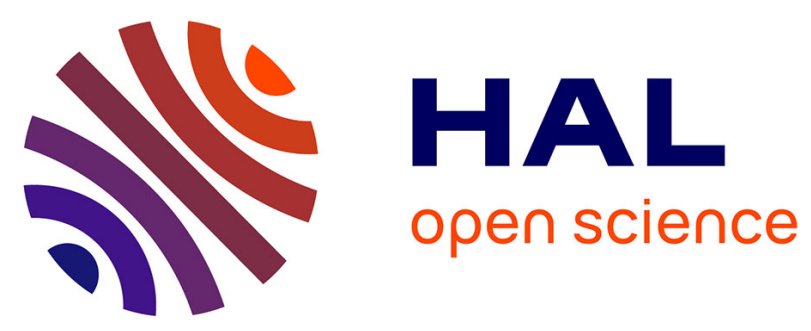

\title{
A Conformal/Rollable Monolithic Miniaturized Ultra-Portable Ground Penetrating Radar Using Additive and Inkjet Printing
}

Anya Traille, Sangkil Kim, Anthony Coustou, Hervé Aubert, Manos Tentzeris

\section{- To cite this version:}

Anya Traille, Sangkil Kim, Anthony Coustou, Hervé Aubert, Manos Tentzeris. A Conformal/Rollable Monolithic Miniaturized Ultra-Portable Ground Penetrating Radar Using Additive and Inkjet Printing. International Microwave Symposium, Jun 2014, Tampa Bay, United States. 4p. hal-01063310

\author{
HAL Id: hal-01063310 \\ https://hal.science/hal-01063310
}

Submitted on 11 Sep 2014

HAL is a multi-disciplinary open access archive for the deposit and dissemination of scientific research documents, whether they are published or not. The documents may come from teaching and research institutions in France or abroad, or from public or private research centers.
L'archive ouverte pluridisciplinaire HAL, est destinée au dépôt et à la diffusion de documents scientifiques de niveau recherche, publiés ou non, émanant des établissements d'enseignement et de recherche français ou étrangers, des laboratoires publics ou privés. 


\title{
A Conformal/Rollable Monolithic Miniaturized Ultra-Portable Ground Penetrating Radar Using Additive and Inkjet Printing
}

\author{
Anya Traille ${ }^{1}$, Sangkil Kim ${ }^{2}$, Antony Coustou ${ }^{1}$, Herve Aubert ${ }^{1}$, Manos M. Tentzeris ${ }^{2}$ \\ ${ }^{1}$ University of Toulouse, INP, LAAS-CNRS, 7 Avenue du Colonel Roche, Toulouse, 31400, France \\ ${ }^{2}$ Georgia Institure of Technology, Atlanta, GA, 30305, USA \\ atraille@laas.fr
}

\begin{abstract}
Typical UWB FMCW Ground Penetrating (GPR) Radars operate at low frequencies that require a wide sweep bandwidth thus necessitating complex architectures and bulky broad-band antennas. This poses unique challenges to the system portability especially for manual, wide-area outdoor measurements. In this paper, we present the first design, fabrication and characterization of a complete conformal and miniaturized radar system to be rolled up in a "poster-like" container using additive printing technology. As the lumped or distributed passives, the active devices and the $R x / T x$ antennas may share the same flexible substrate, the proposed radar technology is considered to be monolithic. The presented proofof-concept system performs the most fundamental operations of the FMCW radar including signal generation, as well as the amplification and correlation of the LO and RF signals for GPR frequencies. Specifically, this paper outlines an ultra low cost system integration, packaging and experimental verification of a flexible/conformal monolithic radar system with almost identical performance for different degrees of flexing.

Index Terms - Monolithic, conformal, FMCW radar, Ground Penetrating Radar, flexible electronics, inkjet printing additive printing.
\end{abstract}

\section{INTRODUCTION}

Radar is a very mature and established technology dating back to the 1920s. FMCW (frequency modulated continuous wave) radar, in particular, combines the advantages of the high-resolution impulse radar $(\Delta \mathrm{R} \sim 1 / \mathrm{B})$ and the low power $\mathrm{CW}$ (continuous wave) radar $\left(\mathrm{E}=\mathrm{P}_{\mathrm{av}} \tau_{\mathrm{p}}\right)$. Other advantages include high SNR, wide dynamic range, low NF, better angular resolution, cheaper/simple low-power components (Power Amplifier and low speed Analog to Digital Converter), and frequency control/versatility, while its main disadvantages include licensing restrictions, and time gating against reflections and multipath. Both Pulsed and FMCW imaging radars have become popular in ground penetrating radar applications with Pulsed radar being more commercially available and the FMCW radar developed and used mainly by research groups.

Advancements in FMCW radar have been mostly due to major hardware developments, signal processing techniques, waveform design, and system level integration of new components into new architectures, such as the Ultra Wideband FMCW Ground Penetrating Radar (GPR) [1] which is a recently developed technology for high resolution imaging applications such as concealed weapon detection and detailed subsurface imaging. In the case of GPR, the unique challenge of Ultra wideband FMCW radar is that it requires the use of very low frequencies $(<300 \mathrm{MHz})$ to obtain penetration depths of at least several meters (to detect ground water for example) and a very wide sweep bandwidth to achieve submeter resolution for detailed subsurface images [1]. This makes typical GPR systems too bulky for backpack transportation and simple deployment. In addition, GPR, unlike other radar systems must perform manual wide-area measurements commonly using a vehicle, sled or by walking. There is a severe lack of flexible radar platforms providing the mechanical versatility that would facilitate geophysical studies (particularly in rugged environments such as the arctic, desert, uneven mountainous terrains or space).

In this paper we propose the first additive-printing-based packaging process of a conformal, monolithic radar system for a UWB FMCW applications operating in the VHF/UHF range for deep/shallow subsurface imaging. The main challenges of packaging a UWB FMCW radar are due to the facts that: (1) the high percentage bandwidth at low frequencies complicates the pure, linear, and spurious-free signal generation (for example limited availability steep-skirted filters) and the receiver requirements therefore requiring very complex receiver architectures [2] and, (2) at low GPR frequencies, very large broadband antennas are required which are commonly bulky.

Such a challenge can be met only with packaging modifications in both the electronic system and the antenna, where they both get combined into one single flexible, conformal, rollable and robust system using a cost effective approach. To achieve that in this paper we had to address the most current challenges in flexible electronics which are related to fabrication (metal thickness uniformity, flexible interconnects, accurate alignment), performance distortion, robustness (delamination of IC's) and lifetime (power handling). Flexible PCBs made from flexible polymer do exist however they still require photolithography and lamination fabrication processes [3]. Cost effective inkjet printing of individual components such as passives, interconnects and antennas for sensor modules has been demonstrated in [4], however this is the first reported design and validation of a complete radar system which can be easily extended to multilayer densely interconnected circuits for more complex multi-mode/multi-channel radar architectures for other radar imaging applications and frequencies. The full 


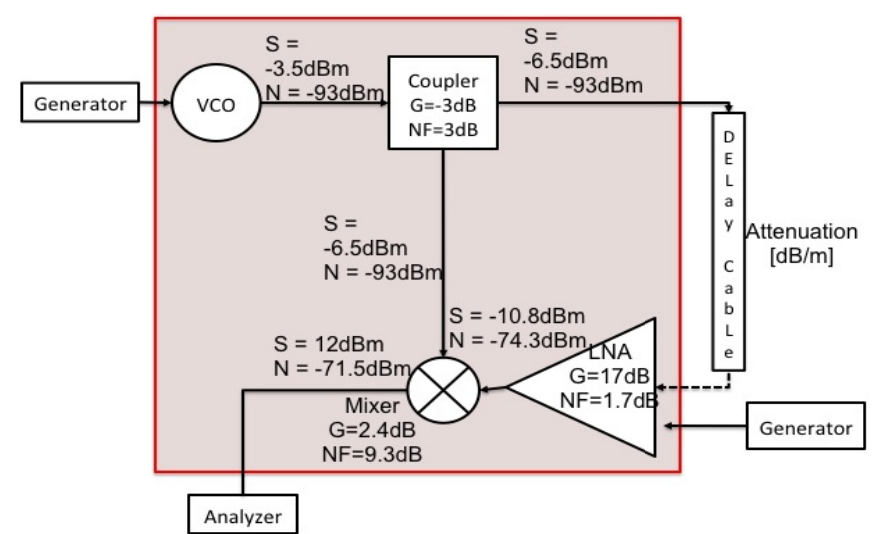

Fig. 1. Link budget estimation of flexible radar system ( $\mathrm{S}=$ signal, $\mathrm{N}=$ noise).

system is referred to as monolithic as lumped or distributed passives (hybrid coupler, matching baluns, filters and mixer), active devices (VCO, LNA and Power Amplifier) and the $\mathrm{Rx} / \mathrm{Tx}$ antennas may share here the same flexible substrate in the proposed radar technology.

The following sections will show the design, fabrication, and measurement of the Monolithic FMCW Ground Penetrating Radar and access the challenges of metal thickness uniformity, IC delamination, alignment and flexible interconnects. Upon completion, the entire system including the antenna is to be presented at the conference.

\section{MONOLITHIC FMCW RADAR DESIGN}

A simple FMCW architecture using operating specifications customized for high resolution GPR was selected to be implemented in Polyethylene terephthalate (PET) film as a proof-of-concept demonstrator. Based on the IF bandwidth and required output SNR (as well as other operating point

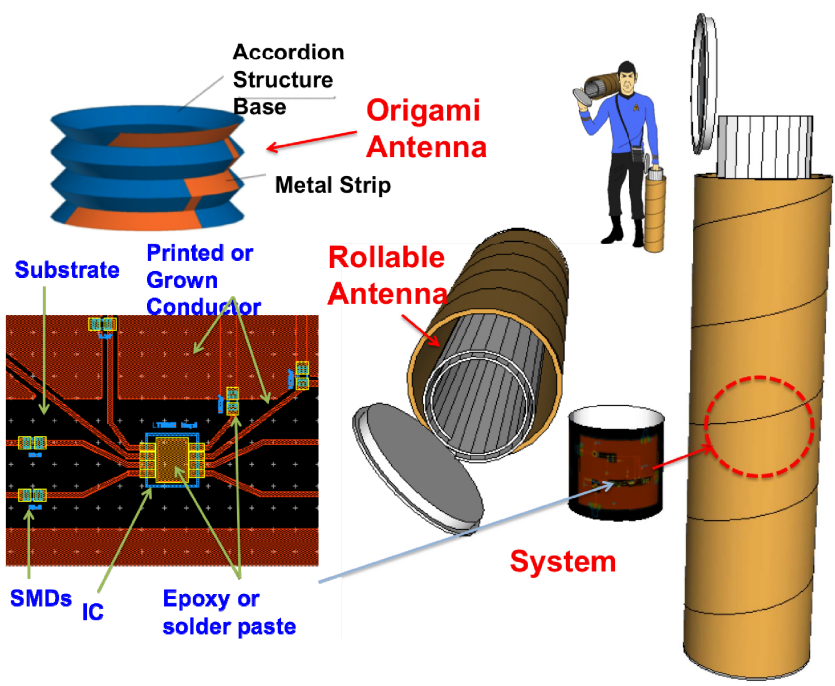

Fig. 2. The left shows a portion of the flexible radar footprint in orange, the right shows the integration into the concept of a portable/conformal FMCW Ground Penetrating Radar designed to be easily attached onto a backpack and quickly unpacked for measurements. specifications) for the FMCW GPR operating from 200$400 \mathrm{MHz}$, the receiver sensitivity was calculated to be around $77 \mathrm{dBm}$. The initial architecture contains the necessary components for signal generation, channel transmission and reception (for initial 'in-cable' testing), amplification and mixing. Fig 1 shows the test architecture and estimated link budget.

The test setup consists of a signal generator that tunes the Voltage Controlled Oscillator (specified output power between -2.5 to $+9.5 \mathrm{dBm}$ but reduced due to harmonic outside test frequency range), which then produces the necessary LO (Local Oscillator) and RF. The Low Noise Amplifier at the RF Input port receives the $\mathrm{RF}$ either from a delay cable or a signal generator, as it happened in this paper. The VCO was chosen appropriately for the desired sweep frequency range and the mixer's required input LO drive power $(<1 \mathrm{dBm})$. A limited number of LNAs for our particular frequency and package specifications were available so we chose the best candidate to reduce mixer noise (typically around $7 \mathrm{dBm}$ but varies) in receiver chain.

The circuit footprints for each component and the traces for the entire system were designed in CadSoft EAGLE as shown in Fig. 2, which clearly demonstrates its rollable/portable feature.

\section{FABRICATION}

The passive interconnects were fabricated using the twostep electroless plating bath process [5], which consists of the inkjet printing and curing of catalytic ink onto PET substrate $\left(\varepsilon_{\mathrm{r}}=2.0, \tan \delta=0.04, \mathrm{t}=0.1 \sim 0.254 \mathrm{~mm}\right.$ followed by electroless growth of the metal $\mathrm{Cu}$ film $(\sigma=3.2 \mathrm{e} 6 \mathrm{~S} / \mathrm{m}$, thickness $=0.61 \sim 3.8 \mu \mathrm{m}$ ).

The Inkjet printing of the catalyst-based ink for electroless plating was chosen for the metallization method in order to improve the flexibility and robustness [4]. The surface mount electronics including ICs and passive lump components are soldered on the circuit footprint utilizing a solder paste for low temperature curing. The soldered components cannot be cracked unless the stresses are applied to the solder directly. This is one out of two fabrication choices, with the other being

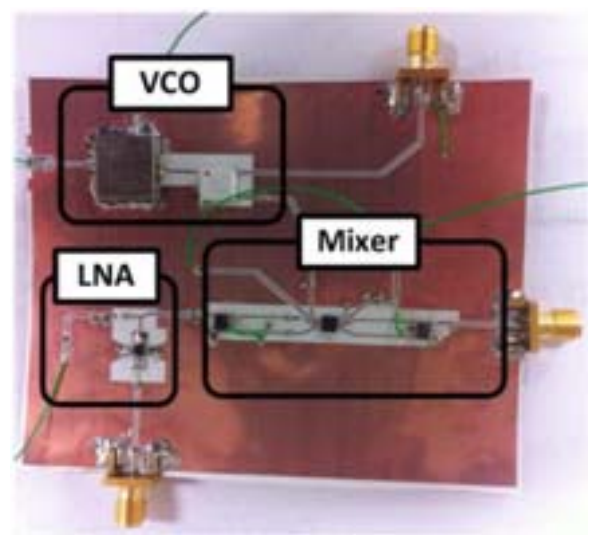

Fig. 3. Part of the fabricated monolithic FMCW radar system with components mounted. 


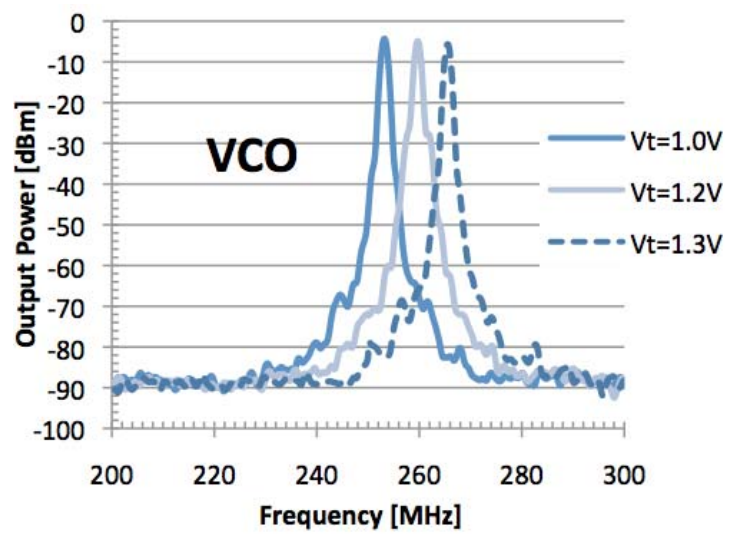

Fig. 4. The measured VCO output (from RF output port) for various tuning voltages $\mathrm{Vt}$

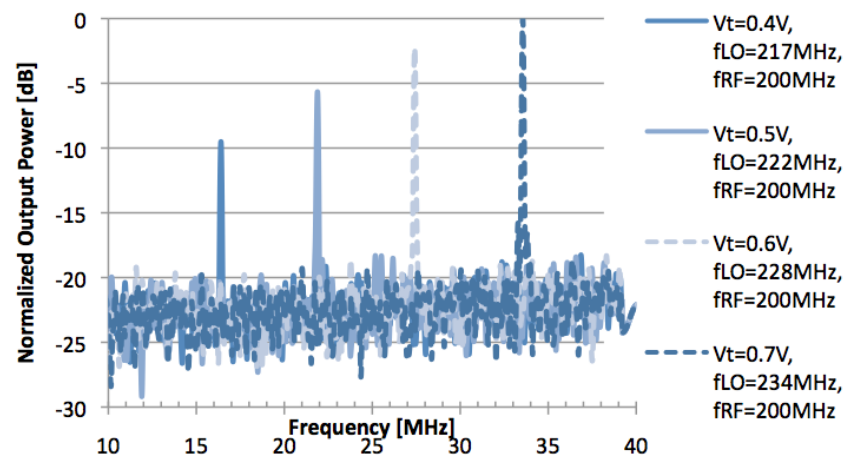

Fig. 5. Normalized IF Output power of the complete system.

the inkjet printing of a silver nanoparticle ink $(\sigma=1.1 \mathrm{e} 7 \mathrm{~S} / \mathrm{m})$ onto paper, PET or LCP $\left(\varepsilon_{\mathrm{r}}=3 \sim 3.2, \tan \delta=0.02 \sim 0.05\right.$, thickness $=0.254 \mathrm{~mm}$ ) which offers a more expensive but improved electrical properties. The fabricated system is shown in Fig. 3.

\section{MEASUREMENTS}

Measurements were performed on the fabricated structure to validate its performance. The signal generated from the Crystek VCO (frequency range of $200 \sim 400 \mathrm{MHz}$, tuning voltage $0 \sim 5 \mathrm{~V}$ was tested at the RF output port of the flexible system (Fig. 4).

Due to the absence of a PLL circuit to stabilize the output frequency control phase noise, the VCO needed to be characterized extensively. The VCO output was measured at the RF output port following the hybrid coupler. The full radar system was measured, starting from signal generation all the way to the IF output port (VCO, Hybrid Coupler, LNA and Mixer). The measured IF output frequency (Fig. 5) corresponded exactly to the RF-LO frequency difference $s$ of $22 \mathrm{MHz}, 28 \mathrm{MHz}$ and $34 \mathrm{MHz}$. All components were confirmed to work exactly as specified. The only system weakness observed was the high insertion loss from the mixer. This could be attributed to the matching circuit. The output power level can be improved by adding an external LC matching network such as a $\mathrm{T}$ - or $-\pi$ matching networks at the
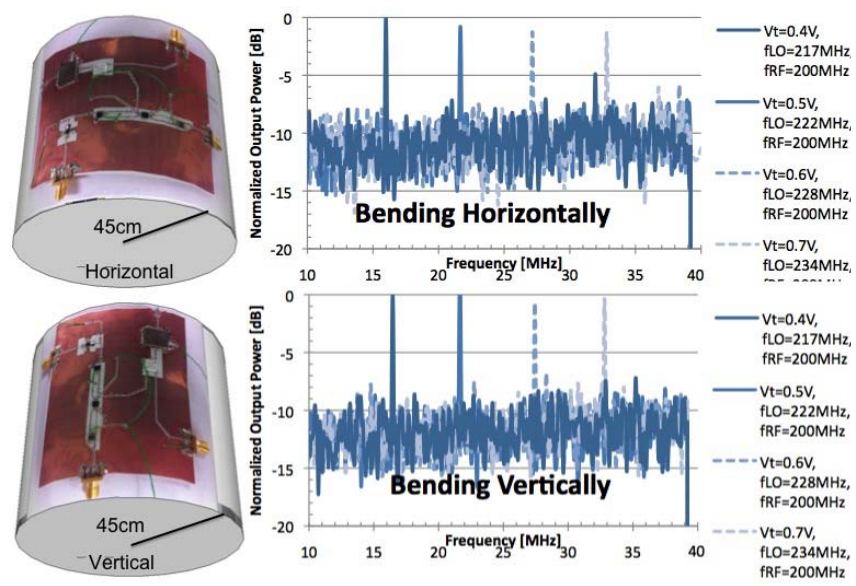

Fig. 6. Measured IF Output of system bent horizontal and vertical.

output port, as such topologies provide more design versatility than $\mathrm{L}$ matching networks. In addition, adjusting the two input power levels (LO and RFin) of the mixer (using attenuators) to be closer together may improve the mixer output signal gain.Due to the absence of a VGA equipped Automatic Gain Control circuit found in many transmitters [1], the normalized output power level changes with frequency.

\section{A. Flexibility Test}

A flexibility test was performed in order to benchmark the system performance in a rolled/flexed state. Fig 6 shows the IF output when wrapping the system around a foam cylinder of radius $4.5 \mathrm{~cm}$ both horizontally and vertically demonstrating an almost flex-independent performance of the radar module. It has to be stressed that output power level (between -70 and $75 \mathrm{dBm}$ in our preliminary experiment) was the same for both rolled and unrolled configurations.

The only parameter limiting the minimum radius of the circuit's bending curvature is limited by the largest surface mount ICs. The largest electronic component of the proposed circuit is the VCO and the whole system (100mm x $80 \mathrm{~mm}$ ) can be easily wrapped around the cylinder of radius is $4.5 \mathrm{~cm}$ without damaging the circuit due to delamination. The inkjetprinted catalyst-based electroless plating improves the flexibility of interconnects because the adhesion of the electroless plated conductor to the substrate is much stronger than printing of nanoparticle-based ink [5].

\section{CONCLUSION}

The first conformal and rollable monolithic radar system has been demonstrated featuring an almost identical performance for rolled/unrolled states. The choice of wide band antenna will be the next step of this work. The antenna gain required to achieve a particular penetration depth must be determined based on the estimated receiver sensitivity and power budget calculations for maximum transmit power 
without exceeding emission limits. Although we are planning to test antennas such as the cone, bowtie, Vivaldi, horn and spiral are recommended for GPR applications [6], we will focus on the integration with inkjet-printed origami-based "accordion" antennas that have recently led to 1-2 orders of size miniaturization and full integrability with inkjet-printed electronic platforms. The simplicity as well as the multilayer/multimaterial capability of the proposed additive printing (fully printed passive, diodes, vias) combined with flexible mounting would enable the realization of highly dense interconnects as well as enhanced miniaturization and conformability of practical GPR systems of the future, that could find numerous applications in wearable biomonitoring, smart skins and geodetection systems.

\section{ACKNOWLEDGEMENT}

It is wished to acknowledge IRD (Institut de Recherche pour le Développement) for supporting the research and development of a new FMCW radar as well as the support of National Science Foundation (NSF).

\section{REFERENCES}

[1] B. Holt, P. Kanagaratnam, S. Gogineni, V. Ramasami, A. Mahoney, and V. Lytle, "Sea ice thickness measurements by ultrawideband penetrating radar: First results," Cold Reg. Sci. Technol., vol. 55, no. 1, pp. 33-46, Jan. 2008.

[2] M. Jankiraman, B. Wessels, and P. van Genderen, "Pandora multifrequency FMCW/SFCW radar," 2000 IEEE International Radar Conference, pp.750-757, May 2000.

[3] [Online] Available: http://www.flexiblecircuit.com/documents/Design-Guide.pdf .

[4] S. Kim, B. Cook, T. Le, J. Cooper, H. Lee, V. Lakafosis, R. Vyas, R. Moro, M. Bozzi, A. Georgiadis, A. Collado and M. M. Tentzeris, "Inkjet-printed Antennas, Sensors and Circuits on Paper Substrate," IET Microw. Antennas Propag., vol.7, no.10, pp.858-868, July 2013.

[5] B. S. Cook, Y. Fang, S. Kim, T. Le, W. B. Goodwin, K. H. Sandhage, and M. M. Tentzeris, "Inkjet catalyst printing and electroless copper deposition for low-cost patterned microwave passive devices on paper," Electron. Mater. Lett., vol. 9, no. 5, pp. 669-676, Sep. 2013.

[6] I. Hertl and M. Strý, "UWB Antennas for Ground Penetrating Radar Application," 2007 Applied Electromagnetics and Communications, pp.1-4, Sep. 2007. 